\title{
Liposomes as delivery systems in the prevention and treatment of infectious diseases
}

- Joep J. Bergers, Timo L.M. ten Hagen, Els W.M. van Etten and Irma A.J.M. Bakker-Woudenberg

\section{Introduction}

The prevention and treatment of infectious diseases has been one of the major successes in medicine. Nevertheless, the need for new and improved vaccines remains as great as ever and the search for new antimicrobial agents and intensification of treatment with existing drugs continues because many infections can not be treated adequately with the established drugs and treatment methods.

For almost 200 years vaccines have consisted of whole micro-organisms, killed or live but attenuated. Many of these vaccines have been highly successful, with perhaps the vaccinia virus for smallpox as the most striking example. Several compelling reasons exist, however, for limiting the number of antigens in a vaccine to those which are protective. It may be desirable to avoid the inclusion of genetic material and toxic components of the microorganism. It may be necessary to circumvent the strategy used by the micro-organism to evade the immune system. The search for new vaccines, i.e. those composed of subunits of micro-organisms, either purified from natural sources or produced by recombinant DNA techniques or by chemical synthesis, has been aided by advances in molecular biochemistry and by understanding the factors regulating the immune response. Unfortunately, these subunit vaccines are not, or only weakly, immunogenic in the absence of an immunological adjuvant.

From clinical experience, it is well known that in immunocompromised patients infections are still a major cause of morbidity and mortality. The number of immunocompromised patients is growing and includes patients with malignant diseases treated with intensive chemotherapy, organ and bone marrow transplant recipients receiving immunosuppressive treatment, and patients with the acquired immunodeficiency syndrome (AIDS). One of the factors that contribute to treatment failure is an impaired host defence system unable to provide adequate support for antibiotic therapy. Improvement of the treatment of severe infections in immunocompromised patients is urgently required.

One approach to increase the immunogenicity of purified microbial antigens and to potentiate the therapeutic action of antimicrobial agents is to employ liposomes as vehicles for their delivery [1-3]. Liposomes are membrane-like vesicles consisting of one or more phospholipid bilayers surrounding aqueous compartments (Fig. 1). Hydrophobic agents can be embedded in the lipid bilayers and hydrophilic agents can be entrapped in the internal aqueous space of the liposomes. The charge, rigidity, size, and surface properties of liposomes can be varied and controlled by incorporating different types of lipids and by varying the preparation method. The fate of liposomes as well as the encapsulated agent in the body is primarily dependent on these parameters (charge, rigidity, size and surface
Bergers II ten Hogen TLM von Etten EWM, Bakker Woudenberg IAM. Liposomes as deliven systems in the prevention and treatment of infectious diseases. Phorm Worla S61.1995.17(1).1.11.

Dr. 1. Bergers (correspondence): Department of Clinical Microbiology, Erasmus University, P. O. Box 1738, 3000 DR Rotterdam, the Netherlands. Present address: Department of Biologicals, Laboratony for Medicines and Medical Devices, National Institute of Public Health and Environmental Protection, P.O. Box 1, 3720 BA Bilthoven, the Netherlands T.L.M. ten Hagen, E.W.M. van Etten and

Dr. 1.A.IM, Bakker-Woudenberg, Department of Clinical Microbiology, Erasmus University Rotterdam, the Netherlands

\section{Keywords}

Antibiotics

Antifungal agents

Antigens

Antiprotozoal agents

Antiviral agents

Drug delivery

Infection

Liposomes

Vaccines

\section{Abstract}

Research on the potential application of liposomes in the prevention and treatment of infectious diseases has focussed on improvement of the therapeutic index of antimicrobial drugs and immunomodulators and on stimulation of the immune response to otherwise weak antigens in vaccines composed of purified micro-organism subunits. In this review current approaches in this field are outlined. The Improved therapeutic index of antimicrobial drugs after encapsulation in liposomes is a resuit of enhanced drug delivery to infected tissue or infected cells and/or a reduction of drug toxicity of potentially toxic antibiotics Liposomal encapsulation of immunomodulators that activate macrophages ains at reducing the toxicity of these agents and targeting them to the cells of the mononuclear phagocyte system in order to increase the nonspecific resistance of the host against infections. Studies on the immunogenicily of liposomal antigens have demonstrated that liposomes can potentiate the humoral and cell mediated immunity to a variety of antigens.

Accepted September 1994. 


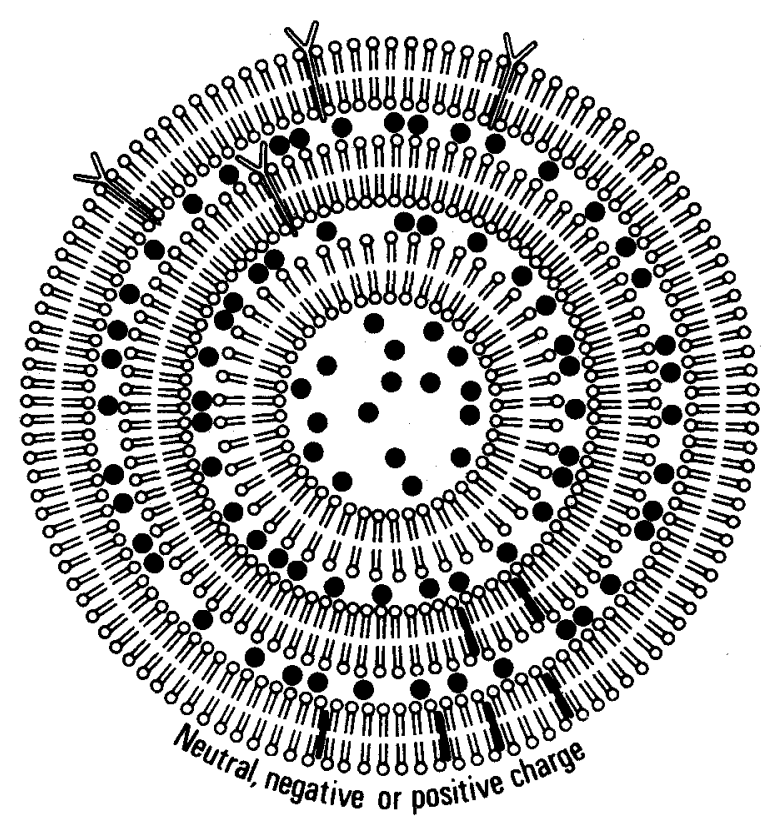

Water soluble molecules
Lipid soluble molecules
Water soluble molecules with hydrophobic
moiety penetrating lipid phase

A Figure 1

Schematic representation of a multilamellar liposome. In an aqueous environment the phospholipids orient themselves in bilayers with their headgroups directed towards the water molecules and the acyl chains

isolated from the water phase. Water-soluble molecules can be entrapped in the internal aqueous space and lipid-soluble molecules can be incorporated in the lipid bilayers. Reprinted with the permission of the New England Journal of Medicine [4]

properties). Therefore, liposomes are considered as versatile delivery systems. The potential widespread clinical use of liposomes, as opposed to many other parenteral drug delivery systems, may result from the fact that the lipids most often used for liposome preparation are those commonly found in cell membranes, and that liposomes are biodegradable, relatively nontoxic, and nonantigenic or weakly antigenic [5 6].

After intravenous administration, the main fraction of almost all types of liposomes is rapidly removed from the blood and taken up by cells of the mononuclear phagocyte system (MPS), particularly the tissue macrophages in liver and spleen [7]. This natural tendency of liposomes to accumulate in the cells of the MPS can be employed for the prevention and treatment of infectious diseases in several ways. Firstly, they can be used as carriers of antibiotics for selective delivery to infections that localize primarily in the cells of the MPS. Increased intracellular antibiotic concentrations may potentiate therapeutic activity by enhancing the delivery of drugs to the intracellular site of infection. Secondly, macrophages may be stimulated into the microbicidal state by liposome-encapsulated immunomodulators for the treatment of both intracellular and extracellular infections. Finally, macrophages play a key role in the induction of immune responses to antigens [8]. The liposome-mediated delivery of antigens to macrophages may be one of the major factors causing liposome-associated antigens to be more immunogenic than the free antigen.

The use of liposomes for the delivery of antibiotics to infections in non-MPS tissues requires a reduced initial uptake by the MPS, enabling the liposomes to circulate long enough to reach the infected tissues or cells. Prolonging the blood residence time of liposomes after intravenous administration can be achieved by manipulation of the physicochemical characteristics of the liposomes. Alternatively, the initial clearance of liposomes by liver and spleen can be avoided by injection of the vesicles into an anatomically defined compartment, which may be of value for local treatment of infections.

The objective of this article is to outline the potential application of liposomes in the field of infectious diseases, that is improvement of the therapeutic index of antimicrobial drugs and immunomodulators and stimulation of the immune response to otherwise weak antigens in vaccines composed of purified micro-organism subunits. As the literature available on this topic is abundant, attention will be focussed on major breakthroughs and recent developments. Unless stated otherwise, liposomes were of the type that after intravenous administration is mainly taken up by cells of the MPS.

\section{Liposome-mediated delivery of antimicrobial agents}

The use of liposomes as antibiotic delivery systems aims for: increased antibiotic concentrations at the site of infection, resulting from targeting the antibiotic to the infected tissues; increased intracellular antibiotic concentrations, resulting from targeting the antibiotic to the infected cells; and reduced toxicity of potentially toxic antibiotics, resulting from avoiding distribution to sites susceptible to toxicity. Application of liposomes may offer an excellent way to increase the selective delivery of antibiotics in these respects. The first studies on the use of liposomes as carriers of antibiotics were performed in models of leishmaniasis and were published in 1978 [9 10]. Until now most experimental data were derived from studies with antibiotic-containing liposomes applied in models of infection by facultative and obligate intracellular pathogens localized in MPS cells. A variety of protozoal, viral, fungal and bacterial infections was studied.

\section{Liposomes for the systemic treatment of} infectious diseases

Due to the natural behaviour of liposomes after intravenous administration the encapsulated antibiotic will be targeted to MPS tissues, resulting in relatively high and prolonged intracellular concentrations of the antibiotic. A poor penetration into the cells and a decreased activity intracellularly are the major reasons for a limited activity of most antibiotics (notably the penicillins, cephalosporins and aminoglycosides) in intracellular infections.

Leishmania are obligate intracellular protozoa residing within macrophages and are harboured inside phagolysosomes. In animal models of leishmaniasis, liposome-encapsulated antimonial compounds and 
liposome-encapsulated amphotericin B (including Amphocil $^{\circledR}$ and AmBisome ${ }^{\circledR}$ ) were applied. In general, all these studies demonstrated an increase in the therapeutic index of the antibiotic (an increase in efficacy as well as a reduction of toxicity of potentially toxic antibiotics) resulting from encapsulation in liposomes [11]. Successful treatment of a patient with drug-resistant visceral leishmaniasis with AmBisome $^{\circledR}$ was reported in 1991 [12]. AmBisome ${ }^{\circledR}$ was also used for the treatment of visceral leishmaniasis in AIDS patients unresponsive to antimonial compounds and appeared to be effective for treatment and secondary prophylaxis [13 14]. In animal models of malaria (Plasmodium berghel), the infection was successfully treated with liposome-encapsulated chloroquine even when the infectious organism was resistant to the free agent [15]. This could be ascribed to sustained release of chloroquine from the intraperitoneally injected liposomes.

With respect to viral infections, it was demonstrated in an animal model of infection caused by Rift Valley fever virus which replicates in liver macrophages, that liposome-encapsulated ribavirin was more effective than the free agent [16]. The data obtained from other animal studies on the efficacy of liposome-encapsulated antiviral agents in various models of viral infection, caused by herpes simplex virus, influenza virus, Rauscher murine leukaemia virus, or murine AIDS, are contradictory with respect to the efficacy of antiviral agent when administered in the liposome-encapsulated form [17-19]. Also data from in vitro studies in which various cell types infected with various viral strains were exposed to antiviral agent in the free form or liposome-encapsulated form are contradictory [19-21]. In some studies antiviral antibody was incorporated into liposomes. As liposomes can be targeted to specific cells by the conjugation of the appropriate antibodies to the lipid bilayer, they are expected to be ideal carriers for the delivery of drugs to virus-infected cells. For immunoliposomes containing aciclovir it was demonstrated that these liposomes specifically bind to herpes simplex virus-infected cells in vitro, and are most efficacious in inhibiting virus replication [22].

Regarding fungal infections, most studies are performed with amphotericin B encapsulated in liposomes or bound to other lipid carriers with the main purpose of reducing the toxicity of this antifungal agent. Extensive studies have been performed with various formulations of liposomal or lipid-complexed amphotericin B [23]. The first study was published in 1982 [24]. Various animal models of fungal infection were used, such as cryptococcosis, histoplasmosis, candidiasis, and aspergillosis to compare the efficacies of free amphotericin versus liposomal or lipidcomplexed amphotericin B [25-29]. Studies were performed in immunocompetent as well as immunodeficient animals. The studies clearly show that, in spite of a lower antifungal activity dependent on the liposome or lipid carrier, due to the decrease of toxicity these amphotericin B formulations display a markedly increased therapeutic index. In the model of systemic candidiasis in immunocompetent or neutropenic mice the efficacy of liposomal amphotericin $B$ bearing anticandidal antibodies was investigated, and was found to be increased compared to identical doses of liposomal amphotericin B [30].
Clinical studies with liposome-encapsulated amphotericin B have been in progress for several years. The first study was published in 1985 by Lopez-Berestein et al. [31], followed by many other publications. Most published clinical data on efficacy are available for AmBisome ${ }^{\circledR}$ showing encouraging results in a variety of fungal infections in immunocompromised patients [32]. With respect to the industrially produced preparations AmBisome ${ }^{\circledR}$, amphotericin B lipid complex (ABLC), and Amphocil ${ }^{\mathbb{B}}$, it is evident that these have quite different structural and pharmacokinetic characteristics [33]. The clinical pharmacokinetics and safety of these formulations have been investigated. It is not clear whether the differences in structure and pharmacokinetics may be important with regard to tolerance and efficacy. Clinical trials comparing these data for the various liposomal and lipid-complexed amphotericin B formulations are needed to assess their clinical value.

In a number of models of intracellular bacterial infection, representative agents of various classes of antibiotics (aminoglycosides, penicillins, cephalosporins, quinolones) were applied in the free or liposome-encapsulated form. Particularly with respect to the aminoglycosides [34], which despite their toxicity still have an important antimicrobial role, the encapsulation into liposomes may reduce the toxicity and, in addition, will change the pharmacokinetics of these agents resulting in a reduction of nephrotoxicity. Bacterial infections were caused by $\mathrm{Myco}$ bacterium tuberculosis, Salmonella spp., Brucella spp., Listeria monocytogenes, and Mycobacterium avium complex [35]. These studies on the treatment of intracellular infections in MPS tissues clearly demonstrate the superior efficacy of administration in the liposome-encapsulated form. In most studies using models of mycobacterial infection in which also the lung was involved in the infection, an increased therapeutic effect of liposome-encapsulated antibiotic over non-encapsulated antibiotic was only observed in the spleen, and not in the lung. However, in some mycobacterial infections the superior effect of liposome-encapsulated antibiotic was also found in the lung. The efficacy of liposomal gentamicin for the treatment of Mycobacterium avium complex infections in AIDS patients is under investigation [36]. For the targeting of liposome-encapsulated antibiotics to the lung, liposomes coated with amylopectin derivatives were employed in an animal model of Legionella pneumophila pneumonia [37]. The antibiotic sisomycin that was encapsulated in these liposomes was highly effective in the treatment of the lung infection as a result of targeting to the infected tissue.

Supplementary to the efficacy data obtained in models of bacterial infection, in vitro data on the role of liposomes in targeting antibiotics to bacterially infected phagocytic cells also show the superiority of liposome-encapsulated antibiotics. In most cases, monocyte or macrophage cultures were infected with bacterial strains such as Staphylococcus aureus, Brucella spp., Salmonella spp., Escherichia coli, Listeria monocytogenes, Chlamydia trachomatis, and Mycobacterium avium complex [35]. Classical liposomes were used. The infected cells were exposed to aminoglycosides, penicillins, cephalosporins or chloramphenicol. Also, studies were performed with 
human monocytes/macrophages infected with Legionella pneumophila or Mycobacterium avium complex, or polymorphonuclear leucocytes of patients with chronic granulomatous disease infected with Staphylococcus aureus. The studies show that the intracellular micro-organisms were effectively killed when the cells were exposed to liposome-encapsulated antibiotic, whereas the same concentration of non-encapsulated antibiotic alone or in combination with placebo liposomes did not show an antimicrobial effect. It was demonstrated that by varying the lipid composition of the liposomes the intracellular degradation of the liposomes could be influenced and thereby the rates at which liposome-encapsulated agents were released and became available to exert their therapeutic action [38].

Animal studies on the treatment of infections by bacterial strains not necessarily located intracellularly in MPS tissues show somewhat contradictory results with respect to the efficacy of liposome-encapsulated antibiotics versus free antibiotics. An explanation for this is that the applicability of liposomes in the treatment of infectious diseases caused by extracellular micro-organisms not restricted to MPS tissues has been strongly limited by the preferential uptake of intravenously administered liposomes by cells of the MPS.

\section{Liposomes avoiding the mononuclear phagocyte system}

The ability to achieve a significantly longer blood residence time of liposomes creates new possibilities for improving the delivery of antibiotics to infected tissues in general, including infections in non-MPS tissues. Many years ago it was already demonstrated that factors contributing to the prolongation of liposome circulation times included the reduction of liposome size, and modification of the liposomal composition yielding an increased rigidity of the bilayer. With such liposomes, administration of relatively high lipid doses is needed to obtain a relatively long circulation time [7]. Later, it was observed that the incorporation of specific glycolipids, such as monosialoganglioside GM-1 and hydrogenated phosphatidylinositol $(\mathrm{HPI})$, resulted in prolonged circulation without the constraint of high lipid doses but application was still limited to the use of rigid bilayers [39 40]. Such liposomes were named MPSavoiding liposomes or Stealth $\left.{ }^{(}\right)$liposomes [41]. Importantly, it was shown that such liposomes exhibit enhanced localization in a variety of implanted tumours in mice [42]. More recently, many reports have shown that the incorporation of phosphatidylethanolamine derivatives of monomethoxy-polyethyleneglycols (PEG-PE) in the liposomal bilayer also enhances the blood circulation time of liposomes to an extent equal to or exceeding that found previously but without the requirements of high lipid dose or rigid nature of the bilayers [39 43-45]. The term sterically stabilized liposomes has been proposed for these PEGylated liposomes.

In our laboratory we investigated the potential of MPS-avoiding liposomes for the enhanced delivery of antibiotics to sites of bacterial infection [46 47]. An experimental model of unilateral pneumonia caused by Klebsiella pneumoniae in rats was used. Bacterial inoculation of the left lung resulted in a progressive
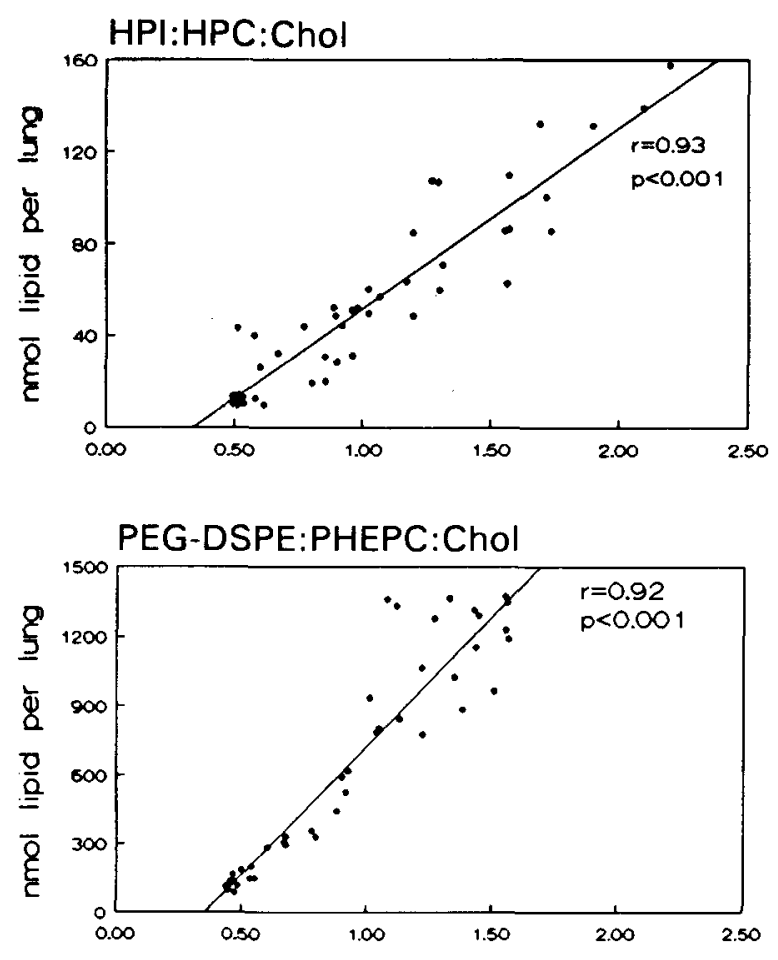

intensity of infection (weight lung in gram)

$\Delta$ Figure 2

Localization of ${ }^{67} \mathrm{Ga}$-desferal labeled liposomes in the lung of rats with Klebsiella pneumoniae lung infection. The increase in weight of infected lung was taken as a measure of the intensity of the infection. Liposomal lipid

(75 $\mu \mathrm{mol} / \mathrm{kg}$ ) was injected intravenously. Localization was determined when $10 \%$ of the liposomal marker was still present in the bloodstream: $16 \mathrm{~h}$ after administration of hydrogenated phosphatidylinositol/ hydrogenated phosphatidylcholine/cholesterol (HPI:HPC:Chol) liposomes (group of 44 rats) and $40 \mathrm{~h}$ after administration of monomethoxypolyethyleneglycol distearoylphosphatidylethanolaminel partially hydrogenated phosphatidy/choline/cholesterol (PEG-DSPE:PHEPC:Chol) liposomes (group of 40 rats). Note the differences in $Y$-axis units

infection of the left lung; the right lung of the same animal developed no infection. Two types of MPSavoiding liposomes, $\mathrm{HPI}$-containing liposomes and PEG-PE-containing liposomes, versus one type of classical (short-circulating) liposomes were examined. Circulation half-lives for HPI-containing liposomes and PEG-PE containing liposomes were $5 \mathrm{~h}$ and $20 \mathrm{~h}$, respectively. The prolonged residence time in the bloodstream was accompanied by a relatively low hepatosplenic uptake.

The degree of lung localization of liposomes was determined in groups of rats with varying intensity of infection. The experimental data, shown in Figure 2, 
indicate that liposomes with prolonged blood circulation time show substantial localization in infected lung tissue after intravenous administration. The PEG-PE-containing liposomes were clearly superior to the HPI-containing liposomes and reached a level of $9 \%$ of the injected liposomal dose in severely infected rats. The increased localization of either liposome preparation in the infected left lung is likely to be related to the local infectious process, as in the right lung of the same rat (in which infection did not develop) the degree of localization of liposomes was not increased compared with the localization observed in uninfected rats. The data suggest that the degree of localization of liposomes in the infected tissue is favoured by a prolonged residence time of liposomes in the blood compartment, enabling the liposomes to reach the infected tissue more efficiently. The degree of lung localization of liposomes correlated with the intensity of the infection, and significantly increased from the moment that bacterial multiplication in the lung resulted in a local inflammatory response. Localization of classical (short-circulating) liposomes in the infected lung tissue was significantly lower.

A valuable asset of the PEG-PE-containing liposomes is that they show dose-independent pharmacokinetics. Prolonged circulation of these liposomes is not dependent on high lipid doses. Therefore, MPS saturation due to high doses of liposomes, which may result in impaired ability to clear bacteria from the blood, can be avoided. This is important as for immunocompromised patients, in which generalization of the infection frequently occurs, maximal blood clearance capacity of the MPS is needed and can be maintained. In addition, the capacity of PEG$\mathrm{PE}$ in prolonging the blood residence time of liposomes has been reported to be independent of the lipid composition (phase transition temperature, charge). In view of the difference in pharmacodynamics of different classes of antibiotics, the rate of release of encapsulated antibiotic from the liposomes is important for an optimum therapeutic effect. Manipulation of the release rate can be achieved by variation in the lipid composition, which in the case of these PEG-PE-containing formulations can be done without compromising the prolonged circulation properties. Therefore, these PEC-PE-containing liposomes show great promise for increased delivery of antibiotics to sites of infection. The factors that account for the extravasation of liposomes in the infected lung tissue have yet to be elucidated. Liposomes probably extravasate in areas of inflammation as a result of locally increased capillary permeability or through injured endothelial linings secondary to the-infectious process. In addition, phagocytosis of liposomes by inflammatory cells infiltrating the infected tissue may also account for enhanced localization of liposomes in the infected lung. The present results derived from the studies in this Klebsiella lung infection model indicate that MPS-avoiding liposomes are 'passively' targeted towards the infected tissue. The passive targeting of these liposomes to the sites of infection is certainly of great importance with respect to clinical application, as in immunocompromised patients the localization as well as the aetiology of the infection is often unknown.

\section{Liposomes for the local treatment of infectious} diseases

The largest number of studies in this field has involved treatment of eye infections, with the aim to increase the availability of the encapsulated antimicrobial agent to the eye in terms of uptake and residence time compared to conventional formulations [48]. The liposomes were administered as eye drops or as subconjunctival or intravitreal injections. Literature dealing with other routes of administration for local treatment of infections is scarce.

Eye drops are easy to use, but the drug tends to be washed away rapidly from the cornea by the tear fluid and by blinking. Consequently, penetration of the drug into ocular tissues is poor and, if penetration occurs, it provides only a short duration of action. Frequent administration is necessary to maintain adequate drug levels.

Smolin et al. demonstrated that a liposomal eye drop formulation containing idoxuridine was more effective for the treatment of experimental herpes simplex keratitis than a comparable therapeutic regime of idoxuridine alone [49]. The authors suggested that the beneficial effects of liposomal administration of idoxuridine were due to improved corneal penetration of the drug and higher therapeutic drug levels in the virus-infected cells, although no direct evidence was presented. Liposomes enhanced the corneal flux and corneal uptake of the hydrophilic antibiotic benzylpenicillin [50], while ocular distribution studies in the rabbit eye indicated that absorption of another hydrophilic antibiotic, dihydrostreptomycin sulfate, was significantly impaired by liposome administration [51]. In conclusion, although some therapeutic results have been reported, the advantage of delivery of liposome-encapsulated antimicrobial agents in eye drop formulations is not clear.

Another approach to obtain a high drug level in the cornea is to inject the drug subconjunctivally. However, the free drug is rapidly removed from the subconjunctival depot, providing limited drug bioavailability. Liposomal gentamicin was found to give higher gentamicin levels in the sclera and cornea up to $24 \mathrm{~h}$ after injection [52]. In a model of keratitis caused by Pseudomonas aeruginosa, a single subconjunctival administration of liposome-encapsulated tobramycin was significantly more effective than a single subconjunctival administration of the free drug [53]. It was nearly as effective as 24 doses of hourly topical fortified tobramycin.

Intravitreal injection of antimicrobial agents has become a part of the standard treatment of some intraocular infections. Unfortunately, in many instances repeated administration is needed to achieve adequate antimicrobial concentrations for prolonged periods of time. Repeated injections, however, can be accompanied by complications, such as lens or retinal injury. Moreover, some antimicrobial agents can cause severe retinal damage. Therefore, liposomes have been explored as depot system, releasing the drug slowly, thereby reducing the toxicity of the drug and maintaining desired drug concentrations for a prolonged period of time. It was demonstrated that gentamicin [54], amikacin [55], ganciclovir [56] and amphotericin B [57] in liposome formulations were cleared from the vitreous signifi- 
cantly more slowly than the free drug. In some cases, therapeutic concentrations of the antimicrobial agent were found in the vitreous for up to two weeks after injection. Residence times of the liposomal antimicrobial agents were dependent on liposome size, liposome composition, and the presence and severity of infection [55 58]. In a model of Staphylococcus aureus endophthalmitis, one injection with liposome-encapsulated clindamycin was demonstrated to eradicate the infection [59]. Unfortunately, the experimental set-up did not include a comparison with the antimicrobial activity of the free drug, thus preventing any conclusion about the effect of encapsulation of the drug. A study of liposomal amphotericin B showed that a reduced toxicity of intravitreally injected liposome-encapsulated amphotericin B was accompanied by a reduced antifungal activity against Candida albicans [60]. No major increase in the therapeutic index of amphotericin B was observed. Preliminary clinical studies indicate that some ocular infections, such as cytomegalovirus retinitis, acute toxoplasmosis, retinochoroiditis and bacterial endophthalmitis, can be managed with intravitreal administration of liposome-encapsulated drugs [56 61].

\section{Liposome-mediated delivery of immunomodulators}

Potentiation of the non-specific defence system with immunomodulating agents to improve the treatment of patients with severe infections is under investigation. The cells of the MPS are generally considered as key cells in the non-specific host defence against infections, and stimulation of these cells by using immunomodulators is of great value for the potentiation of the treatment of severe infections. Especially immunomodulators that exert their major action through macrophages, such as muramyl dipeptide (MDP), the smallest part of the peptidoglycan of the cell wall of bacteria with immunomodulating properties, its amphipathic derivatives, and interferon gamma (IFN $\gamma$ ) have been explored. Although in animal models these agents in the free form enhance the resistance to infections and tumours, their use is hampered by the brief circulation time and poor distribution to the cells of the MPS. Therefore, frequent administration of high doses of these immunomodulators is required to obtain a therapeutic effect. However, this causes severe side effects. As liposomes are taken up preferentially by the cells of the MPS, targeting of immunomodulators to the MPS can be effectively accomplished by administration via liposomes. This so-called passive targeting allows lower dosages to be effective, which favours prolonged treatment protocols. Several studies have shown that administration of immunomodulators in liposomes reduces the toxicity and changes the tissue distribution and pharmacokinetics of the encapsulated agent favourably [62-64]. Moreover, immuno-neutralization and binding to serum proteins is reduced, because of decreased exposure of the agent to the body.

As liposome-encapsulated immunomodulators exert their action mainly via fixed macrophages, the first studies in this field were performed in intracellular infections of the cells of the MPS. It was demonstrated that liposome-encapsulated IFN $\gamma$ was more effective than the free agent in animal models of infection caused by Listeria monocytogenes [65] and Leishmania donovani [64]. These micro-organisms replicate primarily inside macrophages of the MPS. Similar results were reported for free and liposomeencapsulated muramyl tripeptide phosphatidylethanolamide (MTP-PE). In vitro studies with peritoneal macrophages infected with Toxoplasma gondii showed antitoxoplasma activity of liposome-encapsulated IFN $\gamma$ [66]. With respect to suppression of virus replication inside macrophages of the MPS, the efficacy of liposome-encapsulated MTP-PE was studied in an infection model of Rift Valley fever virus [67]. This infection is characterized by multiplication of virus in Kupffer cells and dissemination of virus to the central nervous system. Significant therapeutic effects were observed, whereas free MDP, even at 100 times the dose administered in liposomes, was not effective. Because the monocyte/macrophage serves as a reservoir and vector for HIV, liposomal immunomodulators might also be useful in the treatment of AIDS. In vitro studies have indicated that liposome-encapsulated MTP-PE is a potent inhibitor of HIV replication in macrophages [68]. It should be noted, however, that some viruses show enhanced replication in activated macrophages [69]. Thus, when the antiviral activity of a liposome-encapsulated immunomodulator is studied, it is important to analyse the ability of the virus to replicate in activated macrophages prior to examining in vivo antiviral therapeutic effects.

Exposure of cells in vitro to two or more agents at the same time is easy to achieve. However, in vivo it is unlikely that two agents injected simultaneously will exert their effect on the same cells at the same time. This may be accomplished by the administration of the immunomodulators co-encapsulated in the same liposome, which will result in simultaneous delivery of the agents to the target cell. Treatment of Listeria infection with MTP-PE and IFN $\gamma$ in the same liposome resulted in an enhanced antimicrobial resistance [65]. Synergism between liposomal MTP$\mathrm{PE}$ and IFN $\gamma$ was also shown in a Leishmania infection model [64] and in tumour models [70].

Besides the treatment of intracellular infections of the cells of the MPS with liposomal immunomodulators, extracellular infections and intracellular infections of non-phagocytic cells have been studied. In an experimental model of an infection caused by Klebsiella pneumoniae, a bacterium that is poorly taken up by the cells of the MPS, it was shown that after prophylactic treatment with liposomal MTP-PE or IFN $\gamma$ about $65 \%$ of the mice survived from an otherwise lethal disseminated infection [71]. Administration of MTP-PE and IFN $\gamma$ co-encapsulated in liposomes resulted in an increased efficacy of treatment, with $100 \%$ survival of the infected mice [unpublished observations of our own]. Other investigators showed that the MDP derivative $N$-acetylmuramyl-L$\alpha$-aminobutyryl-D-isoglutamine (Abu-MDP) encapsulated in liposomes is a potent stimulator of the resistance to Candida albicans infections [72].

Macrophages have been shown to play an important role in the control of virus infections of nonphagocytic cells. Activated macrophages acquire the ability to lyse selectively virus-infected cells without harming normal cells. Intravenous administration of 


\section{Table 1 factors affecting the immune response against liposome-associated antigens}

Route of administration

Amount of liposomal antigen administered

Ratio of antigen to lipids

Liposomal antigen localization

Physico-chemical properties of the liposomes:

- lipid composition

- size

- charge

- bilayer rigidity

- surface properties

- lamellarity

Co-encapsulation of immunomodulators employing liposomal immunomodulators, only prophylactic treatment proved to be effective, whereas therapeutic treatment did not show beneficial effects on tumour growth or the course of infection. In one study it even seemed that therapeutic treatment acted negatively on the host defence [71]. When injected in high dosages, liposomes might blockade the phagocytic capacity of macrophages, resulting in a decreased clearance of micro-organisms. Nevertheless, the limited number of animal studies available indicate that prophylactic treatment with liposome-encapsulated immunomodulators might be useful in patients who are at high risk to develop severe infections.

\section{Liposome-mediated delivery of microbial antigens in vaccines}

Aluminum salts are presently the only immunological adjuvant licensed for use in vaccines for humans, but are far from ideal: they do not potentiate cellmediated immunity, they are not always effective and they are not entirely free of undesired reactions at the injection site. The emergence of new-generation recombinant subunit and synthetic peptide vaccines has renewed demands for novel adjuvants that are both acceptable and effective in potentiating humoral as well as cell-mediated immunity.

The first description of liposomes as vehicles of antigens to augment antibody responses appeared in 1974 [81]. Since then, the efficacy of liposomes as vehicles for the presentation of antigens to the immune system has been shown for a variety of bacterial, viral and protozoal antigens. In contrast to many other vehicles, liposome-associated antigens do not induce granulomas at the injection site and hypersensitivity reactions are generally not observed.

Macrophages have been proposed to serve as the predominant antigen-presenting cells in the induction of immune responses by liposomal antigens [2]. After internalization of the liposomes, antigens released from the liposomes are processed in the lysosomal compartment of the macrophage, transported to the cell membrane and presented to $\mathrm{CD}^{+}{ }^{+} \mathrm{T}$ helper cells in the context of major histocompatibility complex (MHC) class II molecules [82 83]. This liposome-mediated delivery of antigens to macrophages may be one of the major factors causing liposomeassociated antigens to be more immunogenic than the free antigens. However, direct interaction of liposomal antigens with lymphocytes and a slow release of antigens from depots at the injection site may play a role as well.

Liposomes are versatile vehicles in terms of structural characteristics and mode of antigen encapsulation. This creates a wide range of options for the design of effective liposomal antigen formulations to induce humoral and cell-mediated immunity. In Table 1 factors are presented that have been shown to affect the immune response to liposome-associated antigens. Such factors may influence the in vivo behaviour of liposomes and their interactions with cells. In spite of much research, considerable controversy surrounds the relationship between liposomal properties and their efficacy as vehicles for antigens. For instance, opposite views have been expressed with regard to the role of liposomal membrane 


\section{Table 2 Microbial antigens* used in vaccine studies}

\section{Bacterial antigens}

Aerobacter levonicum polysaccharide

Bordetella pertussis lipopolysaccharide and outer membrane protein Brucella abortus (lipo)polysaccharide

Mycobacterium leprae soluble antigens

Mycobacterium tuberculosis cell wall subunit antigens

Neisseria gonorrhoeae outer membrane protein

Proteus mirabilis major outer membrane protein and

lipopolysaccharide

Pseudomonas aeruginosa polysaccharide

Salmonella typhimurium polysaccharide

Streptococcus mutans soluble cell wall antigens and carbohydrate protein conjugates

Streptococcus pneumoniae neoglycolipids

Streptococcus sobrinus ribosomal protein

\section{Toxins/toxoids}

Cholera toxin

Diphtheria toxoid

Tetanus toxoid

\section{Protozoal antigens}

Leishmania mexicana surface antigen gp63 and lipophosphoglycan

Leishmania major soluble antigens

Nippostrongylus brasiliensis antigens

Plasmodium falciparum merozoite surface antigens

Plasmadium falciparum sporozoite recombinant protein and synthetic peptide

Toxoplasma gondii membrane protein

\section{Viral antigens}

Adenovirus type 5 hexon and fiber proteins

Encephalomyocarditis virus (inactivated)

Epstein-Barr virus glycoprotein gp 340

Foot-and-mouth virus synthetic peptide

Hepatitis B surface antigen and synthetic peptides

Herpes simplex virus recombinant glycoprotein D and synthetic peptides

Human immunodeficiency virus synthetic peptides

Human T lymphotropic virus hybrid recombinant protein

Influenza virus glycoproteins, reconstituted envelopes and rosettes

Measles virus haemagglutinin and fusion glycoproteins

Parainfluenza virus haemagglutinin-neuraminidase and fusion

glycoproteins

Poliovirus peptides

Rabies virus glycoprotein

Rubella virus rosettes

Semliki Forest virus glycoprotein

Simian immunodeficiency virus synthetic peptide

* Microbial antigens shown are only those of medical or veterinary relevance. Adapted from Gregoriadis [85] and updated. fluidity and antigen localization in promoting immune responses [ 28485$]$. It is likely that the physical and chemical characteristics of liposomes that give optimum responses differ for various antigens. Consequently, characteristics of liposomes would have to be optimized for individual antigens. So far, the approach to design liposomal vaccine formulations and immunization protocols is mainly empirical. No clear insight exists on how to prepare liposome-based vaccines with optimum immunological properties. In addition, little information is available on the efficacy of liposomes as adjuvants in the very young or elderly populations, immunocompromised subjects and genetically low responders or nonresponders.

Class I restricted $\mathrm{CD}^{+}$cytotoxic $\mathrm{T}$ cells $(\mathrm{CTL})$ represent a necessary component for immunity against viral and certain intracellular parasitic infections. Peptides recognized by CTL are generated by the proteolysis of antigens in the cytoplasm and are presented at the cell's surface by MHC class I molecules. Inactivated pathogens and soluble proteins generally fail to prime $C T L$, as these antigens are processed in the endosomes and do not enter the MHC class 1 presentation pathway. Although it was initially thought that liposomes were not very effective in priming $\mathrm{CD}^{+} \mathrm{CTL}$ in vivo [86], recent studies have demonstrated that liposomes containing synthetic peptides or recombinant proteins can induce primary, antiviral CTL responses in animal models [8789]. In these studies modifications of the liposomes, such as coating with a mannan-derivative, inclusion of Quil A or a fusion protein in liposomes, were necessary to deliver the protein into the MHC class I presentation pathway.

In many experimental situations the immunogenicity of the antigen-containing liposomes was still limited. Therefore, immunomodulators were applied as adjuvants to further potentiate the immune response induced by liposome-encapsulated antigen. It is not surprising that amphipathic immunomodulators that activate macrophages have been studied extensively. Monophosphoryl lipid A [90 91] and amphipathic derivatives of MDP [90 92] in particular have been shown to be effective. The best effects of these adjuvants were usually noted when the adjuvant was co-encapsulated with the antigen in the same liposome formulation [93 94]. Recently, much attention has been focussed on the use of cytokines as adjuvants [95 96]. As these agents are rapidly cleared from the circulation, repeated injections are required to obtain strong immune responses. Encapsulation of cytokines in liposomes aims mainly at creating a depot system for sustained release of the cytokine.

Table 2 summarizes many of the microbial antigens encapsulated in liposomes for studying the induction of humoral and cell-mediated immunity in animal models. The antigens employed vary from whole (inactivated) viruses to highly purified antigens obtained by recombinant DNA techniques and synthetically prepared peptides containing $B$ and/or $T$ cell epitopes. These antigens were encapsulated in liposomes or covalently attached to the vesicles. In some studies, the liposomal antigen formulation contained immunomodulators as well. In general, primary and secondary humoral immune responses were 
increased after encapsulation of the antigens in liposomes for all protein antigens tested for humoral immunity. Interestingly, in several studies it was shown that oral or intranasal administration of antigens in liposomes resulted in local secretory IgA levels several times higher than was elicited by free antigen, probably because of vesicle interaction with lymphoid tissue in the gut or bronchus [97 98].

Besides humoral immunity, induction of cellmediated immunity as measured by delayed-type hypersensitivity reactions, lymphocyte proliferation and CTL induction has been reported for several of the liposome-encapsulated antigens summarized in Table 2. In animal models, protection was obtained against infections with Mycobacterium tuberculosis, Salmonella typhimurium, Streptococcus spp., Leishmania spp., Nippostrongylus brasiliensis, Toxoplasma gondii, influenza virus, Epstein-Barr virus, and herpes simplex virus by immunization with liposome-encapsulated antigens [85]. In some of these studies, immunomodulators (MTP-PE, monophosphoryl lipid A) were co-encapsulated with the antigen into the liposomes to induce higher levels of protection. The safety and immunogenicity of a liposome-based malaria vaccine injected into human subjects has recently been reported [99]. High levels of specific antibody were observed.

\section{Conclusions and final remarks}

Animal studies have demonstrated that several intracellular infections involving the MPS can be more effectively treated with liposome-encapsulated antimicrobial agents than with conventional formulations. The natural targeting of liposomes to the phagocytic cells of the MPS results in higher and prolonged antibiotic concentrations at the site of infection. With respect to toxicity, liposomes have proven useful for reducing the toxicity of potentially toxic antimicrobial agents, such as the aminoglycosides and amphotericin B. These promising results of preclinical studies along with technical advances in liposomal preparation techniques and efficient drug entrapment have led to a large number of clinical trials. A liposomal preparation of amphotericin B, AmBisome ${ }^{\circledR}$, was the first liposome preparation licensed on the market in several countries for intravenous application. A number of other lipid-based amphotericin B preparations is likely to follow soon. Additional clinical trials are needed to assess the clinical value of these preparations and to determine the full spectrum of indications.

Although the efficacy of liposomal antibiotics against a number of infections of the MPS has been clearly shown, no systemic efforts have been made to optimize the therapeutic effects. For instance, by manipulating the liposomal composition, rates of uptake and intracellular degradation can be influenced and thereby the rates at which liposomeencapsulated agents are released and become available to exert their therapeutic action.

The recently developed sterically stabilized liposomes open new avenues for achieving specific targeting of antibiotics to infections located outside the tissue macrophages of the liver and spleen. However, at present no studies have been reported on the efficacy of such liposomes in the treatment of microbial infections.

Concerning targeting of immunomodulators to macrophages of the MPS by means of liposomes, experimental evidence is now available of the potential usefulness of liposomes for stimulating the nonspecific antimicrobial resistance. This approach may also be of importance for the potentiation of the treatment of severe infections. Insight into cells and cytokines involved in the enhanced antimicrobial resistance induced by liposome-encapsulated immunomodulators is needed before optimum liposomal dosage forms can be rationally designed.

Liposomes have been shown to act as powerful immunological adjuvants for a variety of bacterial, viral and protozoal antigens, largely through enhanced delivery to antigen-presenting cells. The ability to convert a poorly immunogenic antigen into a highly immunogenic one together with the relatively low toxicity makes liposomes attractive candidates as adjuvants in new generations of vaccines composed of recombinant antigens and synthetically prepared peptides.

\section{References}

1 Swenson CE, Popescu MC, Ginsberg RS. Preparation and use of liposomes in the treatment of microbial infections. Crit Rev Microbiol 1988;15 Suppl 1:1-31.

2 Alving CR. Liposomes as carriers of antigens and adjuvants. I Immunol Methods 1991;140:1-13.

3 Barenholz Y, Crommelin DJA. Liposomes as pharmaceutical dosage forms. In: Swarbrick J, editor. Encyclopedia of pharmaceutical technology. Vol. 9. New York: Marcel Dekker, 1994:1-39.

4 Gregoriadis G. The carrier potential of liposomes in biology and medicine. N Engl I Med 1976;295:704-10.

5 Storm G, Oussoren C. Peeters PAM, Barenholz Y. Tolerability of liposomes in vivo. In: Gregoriadis G, editor. Liposome technology. Vol. III. 2nd ed. Boca Raton: CRC Press, 1993:345-83.

6 Sugerman SM, Perez-Soler R. Liposomes in the treatment of malignancy: a clinical perspective. Crit Rev Oncol Hematol 1992;12:231-42.

7 Senior JH. Fate and behavior of liposomes in vivo: a review of controlling factors. Crit Rev Ther Drug Carrier Syst 1987; 3:123-93.

8 Van Rooijen N. Antigen processing and presentation in vivo: the microenvironment as a crucial factor. Immunol Today 1990;11:436-9.

9 Alving CR, Steck EA, Chapman WL, Waits VB, Hendricks LD, Swartz GM, Hanson WL. Therapy of leishmaniasis: superior efficacies of liposome-encapsulated drugs. Proc Natl Acad Sci USA 1978;75:2959-63.

10 New RRC, Chance ML, Thomas SC, Peters W. Antileishmanial activity of antimonials entrapped in liposomes. Nature 1978;272:55-6.

11 Croft SL. Liposomes in the treatment of parasitic diseases. Pharm Int 1986;7:229-33.

12 Davidson RN, Croft SL, Scott A, Maini M, Moody AH, Bryceson ADM. Liposomal amphotericin $B$ in drug-resistant visceral leishmaniasis. Lancet 1991;337:1061-2.

13 Torre-Cisneros J, Villanueva JL, Kindelan JM, Jurado R, Sanchez-Guijo P. Successful treatment of antimony-resistant visceral leishmaniasis with liposomal amphotericin B in patients infected with human immunodeficiency virus. Clin Infect Dis 1993;17:625-7.

14 Lazanas MC, Tsekes GA, Papandreou S, Harhalakis N,

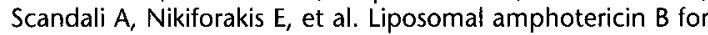
leishmaniasis treatment of AIDS patients unresponsive to antimonium compounds. AIDS 1993;7:1018-9.

15 Peeters PAM, Huiskamp CWEM, Eling WMC, Crommelin DJA. Chloroquine containing liposomes in the chemotherapy of murine malaria. Parasitology 1989;98:381-6.

16 Kende M, Alving CR, Rill WL, Swartz GM, Canonico PG. Enhanced efficacy of liposome-encapsulated ribavirin against Rift Valley fever virus infection in mice. Antimicrob Agents Chemother 1985;27:903-7.

17 Gangemi JD, Nachtigal M, Barnhart D, Krech L, Jani P. Therapeutic efficacy of liposome-encapsulated ribavirin and 
muramyl tripeptide in experimental infection with influenza or herpes simplex virus. I Infect Dis 1987;155:510-7.

18 Phillips NC, Tsoukas C. Liposomal encapsulation of azidothymidine results in decreased hematopoietic toxicity and enhanced activity against murine acquired immunodeficiency syndrome. Blood 1992;79:1137-43.

19 Chu Cl, Szoka FC. Antiviral activity and pharmacokinetics of liposome-encapsulated phosphonoformate in Rauscher murine leukemia virus-infected mice. I Liposome Res 1992;2: $67-92$.

20 Szoka FC, Chu C]. Increased efficacy of phosphonoformate and phosphonoacetate inhibition of herpes simplex virus type 2 replication by encapsulation in liposomes. Antimicrob Agents Chemother 1988;32:858-64.

21 Wyde PR, Six HR, Wilson SZ, Gilbert BE, Knight V. Activity against rhinoviruses, toxicity, and delivery in aerosol of enviroxime in liposomes. Antimicrob Agents Chemother 1988;32:890-5

22 Norley SG, Huang L, Rouse BT. Targeting of drug loaded immunoliposomes to herpes simplex virus infected corneal cells: an effective means of inhibiting virus replication in vitro. I Immunol 1986;136:681-5.

23 Gates C, Pinney RI. Amphotericin B and its delivery by liposomal and lipid formulations. J Clin Pharm Ther 1993;18: 147-53.

24 Graybill JR, Craven PC, Taylor RL, Williams DM, Magee WE. Treatment of murine cryptococcosis with liposome associated amphotericin B. J Infect Dis 1982;145:748-52.

25 Lopez-Berestein G, Hopfer RL, Mehta R, Mehta K, Hersh EM, Juliano RL. Liposome-encapsulated amphotericin B for treatment of disseminated candidiasis in neutropenic mice. Infect Dis 1984;150:278-83.

26 Clark IM, Whitney RR, Olsen SI, George RI, Swerdel MR, Kuuselman L, et al. Amphotericin B lipid complex therapy of experimental fungal infections in mice. Antimicrob Agents Chemother 1991;35:615-21.

27 Adler-Moore JP, Chiang SM, Satorius A, Guerra D, MC Andrews B, Mc Manus EJ, et al. Treatment of murine candidosis and cryptococcosis with unilamellar liposomal amphotericin B formulation (AmBisome). I Antimicrob Chemother 1991;28 Suppl B:63-71.

28 Clemons KV, Stevens DA. Comparative efficacy of amphotericin B colloidal dispersion and amphotericin B deoxychol ate suspension in treatment of murine coccidioidomycosis. Antimicrob Agents Chemother 1991;35:1829-33

29 Van Etten EWM, van den Heuvel-de Groot C, BakkerWoudenberg IAIM. Efficacies of amphotericin B-desoxycholate (Fungizone), liposomal amphotericin B (AmBisome), and fluconazole in the treatment of systemic candidosis in immunocompetent and leukopenic mice. Antimicrob Chemother 1993:32:723-39.

30 Hospenthal DR, Rogers AL, Beneke ES. Effect of attachment of anticandidal antibody to the surfaces of liposomes encapsulating amphotericin B in the treatment of murine candidiasis. Antimicrob Agents Chemother 1989;33:16-8.

31 Lopez-Berestein G, Fainstein V, Hopfer R, Mehta K, Sullivan MP, Keating $M$, et al. Liposomal amphotericin B for the treatment of systemic fungal infections in patients with cancer: a preliminary study. J Infect Dis 1985;151:704-10.

32 Chopra R, Fielding A, Goldstone AH. Successful treatment of fungal infections in neutropenic patients with liposomal amphotericin B (AmBisome). A report on 40 cases from a single center. Leuk Lymphoma 1992;7(Suppl):73-7.

33 lanknegt $R$ de Marie S, Bakker-Woudenberg IAIM Crommelin D|A. Liposomal and lipid formulations of am photericin B. Clinical pharmacokinetics. Clin Pharmacokinet 1992:23:279-91

34 Karlowsky |A, Zhanel GG. Concepts on the use of liposomal antimicrobial agents: applications for aminoglycosides. Clin infect Dis 1992; 15:654-67.

35 Bakker-Woudenberg IAIM, Lokerse AF, ten Kate MT, Melissen PMB, van Vianen W, van Etten EWM. Liposomes as carriers of antimicrobial agents on immunomodulatory agents in the treatment of infections. Eur \& Clin Microbio Infect Dis 1993;12 Suppl 1:61-7.

36 Nightingale SD, Saletan SL, Swenson CE, Lawrence AJ, Watson DA, Pilkiewicz FG, et al. Liposome-encapsulated gentamicin treatment of Mycobacterium avium-Mycobac terium intracellulare complex bacteremia in AIDS patients. Antimicrob Agents Chemother 1993;37:1869-72

37 Sunamoto I, Goto M, lida T, Hara K, Saito A, Tomonaga A Unexpected tissue distribution of liposomes coated with amylopectin derivatives and successful use in the treatment of experimental Legionaires' diseases. In: Gregoriadis $C_{\text {, }}$ Poste G, Senior I, Trouet A, editors. Receptor-mediated targeting of drugs. New York: Plenum Press, 1983:359-66.

38 Bakker-Woudenberg IAIM, Lokerse AF, Roerdink FH. Antibac terial activity of liposome-entrapped ampicillin in vitro and in vivo in relation to the lipid composition. I Pharmacol Exp
Ther 1989;251:321-7

39. Allen TM. Stealth liposomes: five years on. I Liposome Res 1992;2:289-305.

40 Gabizon A, Papahadjopoulos D. The role of surface charge and hydrophilic groups on liposome clearance in vivo. Biochim Biophys Acta 1992;1103:94-100.

1 Allen TM. Stealth liposomes: avoiding reticuloendothelial uptake. In: Lopez-Berestein G, Fidler I, editors. Liposomes in the therapy of infectious diseases and cancer. New Series, 89. New York: Alan R. Liss, 1989:405-15.

42 Gabizon A, Price DC, Huberty J, Bresalier RS, Papahadjopoulos D. Effect of liposome composition and other factors on the targeting of liposomes to experimental tumors: biodistribution and imaging studies. Cancer Res 1990;50:6371.

43 Allen TM, Hansen C. Pharmacokinetics of stealth versus conventional liposomes: effect of dose. Biochim Biophys Acta 1991;1068:133-41.

44 Woodle MC, Matthay KK, Newman MS, Hidayat IE, Collins $L R$, Redemann $C$, et al. Versatility in lipid compositions showing prolonged circulation with sterically stabilized liposomes. Biochim Biophys Acta 1992;1105:193-200.

45 Woodle MC, Lasic DD. Sterically stabilized liposomes. Biochim Biophys Acta 1992;1113:171-99.

46 Bakker-Woudenberg IAIM, Lokerse AF, ten Kate MT, Storm C. Enhanced localization of liposomes with prolonged blood circulation time in infected lung tissue. Biochim Biophys Acta 1992;1138:318-26.

47 Bakker-Woudenberg IAIM, Lokerse AF, ten Kate MT, Mouton IW, Woodle MC, Storm G. Liposomes with prolonged blood circulation and selective localization in Klebsiella pneumoniae-infected lung tissue. J Infect Dis 1993;168:164-71.

48 Niesman MR. The use of liposomes as drug carriers in ophthalmology. Crit Rev Ther Drug Carrier Syst 1992;9:1-38.

49 Smolin D, Okumoto $M$, Feiler S, Condon D. Idoxuridineliposome therapy for herpes simplex keratitis. Am J Ophthalmol 1981:91:220-5.

50 Schaeffer HE, Krohn D. Liposomes in topical drug delivery. Invest Ophthalmol Vis Sci 1982;22:220-7

51 Singh $K$, Mezei M. Liposomal ophthalmic drug delivery system. II. Dihydrostreptomycin sulphate. Int / Pharmaceutics $1984 ; 19: 263-9$

52 Barza M, Baum I, Szoka F. Pharmacokinetics of subconjunctival liposome-encapsulated gentamicin in normal rabbit eyes. Invest Ophthalmol Vis Sci 1984;25:486-90.

53 Assil KK, Frucht-Perry \& Ziegler E, Schnanzlin D), Schneiderman T, Weinreb RN. Tobramycin liposomes, single subconjunctival therapy of pseudomonal keratitis. Invest Ophthalmol Vis Sci 1991;32:3216-20

54 Fishman PH, Peyman GA, Lesar T. Intravitreal liposomeencapsulated gentamicin in a rabbit model. Invest Ophthalmol Vis Sci 1986;27:1103-6.

55 Zeng $\mathrm{S}$, Hu C. Wei $\mathrm{H}$, Lu Y, Zhang $\mathrm{Y}$, Yang ], et al. Intravitreal pharmacokinetics of liposome-encapsulated amikacin in a rabbit model. Ophthalmology 1993;100:1640-4.

56 Díaz-Lopis $M$, Martos MI, España E, Cervera $M$, Vila AO, Navea $A$, et al Liposomally-entrapped ganciclovir for the treatment of cytomegalovirus retinitis in AIDS patients. Doc Ophthalmol 1992;82:297-305.

57 Tremblay C, Barza M, Szoka F, Lahav M, Baum I. Reduced toxicity of liposome-associated amphotericin B injected intravitreally in rabbits. Invest Ophthalmol Vis Sci 1985;26: $711-8$.

58 Barza M, Stuart M, Szoka F. Effect of size and lipid composition on the pharmacokinetics of intravitreal liposomes. invest Ophthalmol Vis Sci 1987;28:893-900.

59 Rao VS, Peyman GA, Khoobehi B, Vangipuram S. Evaluation of liposome-encapsulated clindamycin in Staphylococcus aureus endophthalmitis. Int Ophthalmol 1989;13:181-5

60 Liu KR, Peyman GA, Khoobehi B. Efficacy of liposome-bound amphotericin $B$ for the treatment of experimental fungal endophthalmitis in rabbits. Invest Ophthalmol Vis Sci 1989; 30:1527-34

61 Peyman GA, Charles HC, Liu KR, Khoobehi B, Niesman M. Intravitreal liposome-encapsulated drugs: a preliminary human report. Int Ophthalmol 1988;12:175-82

62 Fidler IJ, Brown NO, Hart IR. Species variability for toxicity of free and liposome-encapsulated muramyl peptides administered intravenously. J Biol Resp Mod 1985;4:298-309.

63 Schumann GP, van Hoogevest $P$, Fankhauser $P$, Probst $A$, Peck $A$, Court $M$, et al. Comparison of free and liposomal MTPPE: pharmacological, toxicological and pharmacokinetic aspects. In: Lopez-Berestein G, Fidler If, editors. Liposomes in the therapy of infectious diseases and cancer. New Series 89. New York: Alan R. Liss, 1989:191-203.

64 Hockertz S, Franke G, Paulini I, Lohmann-Matthes ML. immunotherapy of murine visceral leishmaniasis with murine recombinant interferon- $\gamma$ and MTP-PE encapsulated in liposomes. J Interferon Res 1991;11:177-85. 
65 Melissen PMB, van Vianen $W$, Bidjai $O$, van Marion $M$, Bakker-Woudenberg IAJM. Free versus liposome-encapsulated muramyl tripeptide phosphatidylethanoiamide (MTPPE) and interferon- $\gamma$ (IFN- $\gamma$ ) in experimental infection with Listeria monocytogenes. Biotherapy 1993;6:113-24.

66 Mellors JW, Debs RI, Ryan IL. Incorporation of recombinant gamma interferon into liposomes enhances its ability to induce peritoneal macrophage antitoxoplasma activity. Infect Immun 1989;57:132-7.

67 Kende M, Schroit Al, Rill W, Canonico PG. Treatment of Rift Valley fever virus-infected swiss mice with liposome-encapsulated lipophilic muramyl dipeptide [abstract]. In: Abstracts of the International Conference on Antimicriobial Agents and Chemotherapy. 1983 Oct 24-26; Las Vegas. Washington DC: American Society for Microbiology, 1983: 108

68 Lazdins JK, Woods-Cook K, Walker M, Alteri E. The lipophilic muramyl peptide MTP-PE is a potent inhibitor of HIV replication in macrophages. AIDS Res Hum Retroviruses 1990; 6:1157-61.

69 Hotta H, Hotta S. Dengue virus multiplication in cultures of mouse peritoneal macrophages: effects of macrophage activators. Microbiol Immunol 1982;26:665-76.

70 Saiki I, Fidler IJ. Synergistic activation by recombinant mouse interferon- $\gamma$ and muramyl dipeptide of tumoricidal properties in mouse macrophages. J Immunol 1985;135:684-8.

71 Melissen PMB, van Vianen W, Bakker-Woudenberg IAIM. Treatment of Klebsiella pneumoniae septicemia in normal and leukopenic mice by liposome-encapsulated muramyl tripeptide phosphatidylethanolamide. Antimicrob Agents Chemother 1994;38:147-50.

72 Eppstein DA, Van Der Pas MA, Fraser-Smith EB, Kurahara CG, Felgner PL, Matthews TR, et al. Liposome-encapsulated muramyl dipeptide analogue enhances non-specific host immunity. Int / Immunother 1986;2:115-26.

73 Gangemi JD, Nachtigal M, Barnhart D, Krech L, Jani P. Therapeutic efficacy of liposome-encapsulated ribavirin and muramyl tripeptide in experimental infection with influenza or herpes simplex virus. J Infect Dis 1987;155:510-7.

74 Koff WC, Showalter SD, Hampar B, Fidler IJ. Protection of mice against fatal herpes simplex type 2 infection by liposomes containing muramyl tripeptide. Science 1985;228: 495-7.

75 Duzgunes N, Perumal VK, Brunette EN, Gangadharam PR, Debs Rj. Treatment of Mycobacterium avium complex infections by free and liposome-encapsulated tumor necrosis factor-alpha (Cachectin): studies on peritoneal macrophages and the beige mouse model. In: Lopez-Berestein G, Fidler I], editors. Liposomes in the therapy of infectious diseases and cancer. New Series 89. New York: Alan R. Liss, 1989:287-94.

76 Sheehan KCF, Schreiber RD. The synergy and antagonism of interferon- $\gamma$ and TNF. In: Beutler B, editor. Tumor necrosis factor, the molecules and their emerging role in medicine. New York: Raven Press, 1992:145-78.

77 Murray JL, Kleinerman ES, Cunningham JE, Tatom JR, Andrejcio K, Lepe-Zuniga J, et al. Phase I trial of liposomal muramyl tripeptide phosphatidylethanolamine in cancer patients. I Clin Oncol 1989:7:1915-25

78 Creaven PJ, Cowens JW, Brenner DE, Dadey BM, Han T, Huben $R$, et al. Initial clinical trial of the macrophage activator muramyl tripeptide-phosphatidylethanolamine encapsulated in liposomes in patients with advanced cancer. / Biol Resp Mod 1990;9:492-8.

79 Kleinerman ES, Fia SF, Griffin I, Seibel NL, Benjamin RS, Jaffe $\mathrm{N}$. Phase II study of liposomal muramyl tripeptide in osteosarcoma: the cytokine cascade and monocyte activation following administration. J Clin Oncol 1992;10:1310-6

80 Liebes L, Walsh CM, Chachoua R, Oratz R, Richards $D$, Hochster $\mathrm{H}$, et al. Modulation of monocyte functions by muramyl tripeptide phosphatidylethanolamine in a phase II study in patients with metastatic melanoma. I Natl Cancer Inst 1992;84:694-9.

81 Allison AC, Gregoriadis G. Liposomes as immunological adjuvants. Nature (London) 1972;252:252.

82 Dal Monte PR, Szoka FC. Effect of liposome encapsulation on antigen presentation in vitro: comparison of presentation by peritoneal macrophages and B cell tumors. J Immunol $1989 ; 142: 1437-43$

83 Alving CR. Immunologic aspects of liposomes: presentation and processing of liposomal protein and phospholipid antigens. Biochim Biophys Acta 1992;1113:307-22.

84 Eppstein DA, Byars NE, Allison AC. New adjuvants for vaccines containing purified protein antigens. Adv Drug Del Rev 1989;4:233-53.

85 Gregoriadis G. Immunological adjuvants: a role for liposomes. Immunol Today 1990;11:89-97.

86 Burakoff SJ, Mescher MF. Reconstituted membranes and liposomes in the study of lymphocyte interaction. Cell Surface Rev 1982;8:173-213.
87 Noguchi $Y$, Noguchi T, Sato T, Yokoo $Y$, Itoh S, Yoshida $M$, et al. Priming for in vitro and in vivo anti-human $T$ lymphotropic virus type 1 cellular immunity by virus-related protein reconstituted into liposome. I Immunol 1991;146:3599-603. 88 Miller MD, Gould-Fogerite S, Shen L, Woods RM, Koenig S Mannino R], et al. Vaccination of rhesus monkeys with synthetic peptide in a fusogenic proteoliposome elicits simian immunodeficiency virus-specific CD8+ cytotoxic T lymphocytes. I Exp Med 1992;176:1739-44.

89 Lipford GB, Wagner $H$, Heeg K. Vaccination with immunodominant peptides encapsulated in Quil A-containing liposomes induces peptide-specific primary CD8+ cytotoxic T cells. Vaccine 1994;12:73-80.

90 Brynestad K, Babbitt B, Huang L, Rouse BT. Influence of peptide acylation, liposome incorporation, and synthetic immunomodulators on the immunogenicity of a 1-23 peptide of glycoprotein D of herpes simplex virus: implications for subunit vaccines. I Virol 1990;64:680-5.

91 Alving, CR. Lipopolysaccharide, lipid A, and liposomes containing lipid $A$ as immunologic adjuvants. Immunobiol 1993; 187:430-46

92 Ho RJY, Burke RL, Merigan TC. Antigen-presenting liposomes are effective in treatment of recurrent herpes simplex virus genitalis in guinea pigs. J Virol 1989;63:2951-8.

93 Naylor PT, Larsen HS, Huang L, Rouse BT. In vivo induction of anti-herpes simplex virus immune response by type 1 antigens and lipid $A$ incorporated into liposomes. Infect Immun 1982;36:1209-16.

94 Zhou F, Huang L. Monophoshoryl lipid A enhances specific CTL induction by a soluble protein antigen entrapped in liposomes. Vaccine 1993;11:1139-44.

95 Mbawuike IN, Wyde PR, Anderson PM. Enhancement of the protective efficacy of inactivated influenza $A$ virus vaccine in aged mice by IL-2 liposomes. Vaccine 1990;8:347-52.

96 Bergers II, den Otter W, Dullens HF), Kerkvliet CTM Crommelin DIA. Interleukin-2-containing liposomes: interaction of interleukin-2 with liposomal bilayers and preliminary studies on application in cancer vaccines. Pharm Res 1993;10:1715-21.

97 Abraham E. Intranasal immunization with bacterial polysaccharide containing liposomes enhances antigen-specific pulmonary secretory antibody response. Vaccine 1992;7: 461-8.

98 Michalek SM, Childers NK, Katz J, Dertzbaugh M, Zhang S, Russell MW, et al. Liposomes and conjugate vaccines for antigen delivery and induction of mucosal immune responses. Adv Exp Med Biol 1992;327:191-8.

99 Fries LF, Gordon DM, Richards RL, Egan JE, Hollingdale MR, Gross $M$, et al. Liposomal malaria vaccine in humans: a safe and potent adjuvant strategy. Proc Natl Acad Sci USA 1992;89:358-62 